\title{
Molecular profiling of glatiramer acetate early treatment effects in multiple sclerosis
}

\author{
Anat Achiron*, Anna Feldman and Michael Gurevich \\ Multiple Sclerosis Center, Sheba Medical Center, Tel-Hashomer, and Sackler School of Medicine, Tel-Aviv \\ University, Israel
}

\begin{abstract}
Background: Glatiramer acetate (GA, Copaxone $\left.{ }^{\circledR}\right)$ has beneficial effects on the clinical course of relapsing-remitting multiple sclerosis (RRMS). However, the exact molecular mechanisms of GA effects are only partially understood.

Objective: To characterized GA molecular effects in RRMS patients within 3 months of treatment by microarray profiling of peripheral blood mononuclear cells (PBMC).

Methods: Gene-expression profiles were determined in RRMS patients before and at 3 months after initiation of GA treatment using Affimetrix (U133A-2) microarrays containing 14,500 well-characterized human genes. Most informative genes (MIGs) of GA-induced biological convergent pathways operating in RRMS were constructed using gene functional annotation, enrichment analysis and pathway reconstruction bioinformatic softwares. Verification at the mRNA and protein level was performed by qRT-PCR and FACS.

Results: GA induced a specific gene expression molecular signature that included altered expression of 480 genes within 3 months of treatment; 262 genes were up-regulated, and 218 genes were down-regulated. The main convergent mechanisms of GA effects were related to antigen-activated apoptosis, inflammation, adhesion, and MHC class-I antigen presentation.

Conclusions: Our findings demonstrate that GA treatment induces alternations of immunomodulatory gene expression patterns that are important for suppression of disease activity already at three months of treatment and can be used as molecular markers of GA activity.
\end{abstract}

Keywords: Multiple sclerosis, gene expression, markers, immune-modulation, Glatiramer acetate

\section{Introduction}

Multiple sclerosis (MS) is a progressive central nervous system demyelinating disease, affecting young adults and leading to progressive disability overtime. The disease develops in genetically predisposed subjects and its pathogenesis involves immunological mechanisms associated with autoreactive recurrent assaults against myelin antigens. Along the disease process, mechanisms leading to inflammation, demyelination, and axonal loss accumulate, defining, at the same time, the biological character of the disease and the targets for optimal therapy $[1,2]$.

*Corresponding author: A. Achiron, MD, PhD, Multiple Sclerosis Center, Sheba Medical Center, Tel-Hashomer, 52621, Israel. Tel.: +972 3 5303811; Fax: +972 3 5348186; E-mail: achiron@post.tau. ac.il.
Glatiramer Acetate (GA, Copaxone), a synthetic amino acid copolymer, is an approved first-line treatment for relapsing-remitting multiple sclerosis (RRMS) patients, and is affective in the suppression of disease activity and progression. GA suppress relapse frequency and decreases the accumulation of supresses disability as well as brain MRI activity by reducing lesion load and brain atrophy $[3,4]$.

An expanding body of biological and clinical studies have been undertaken to clarify the mechanisms by which GA exerts its effects in MS; these studies demonstrated that GA immunomodulates various levels of the immune response, including binding to $\mathrm{MHC}$ class II molecules, induction of specific suppressor $\mathrm{T}$ helper 2 (Th2) cells that migrate to the brain and induce in-situ bystander suppression, and induction of anti-inflammatory cytokine production like IL-10 and TGF- $\beta$ [5]. Additionally, GA was shown to bear indi- 
rect neuroprotective effects as passive transfer of GA specific T-cells increased the expression of neurotrophic factors like brain-derived neurotrophic factor (BDNF) and NT-3, 4 that enhance remyelination and axonal growth [5]. Additionally, recent studies demonstrated that GA influenced the stability of nerve terminals in the spinal cord of animals with experimental autoimmune encephalomyelitis [6-8].

Despite this great progress in understanding GAinduced immunomodulatory machinery in MS, the exact molecular mechanisms as well as biomarkers by which it affects the disease process especially in the early treatment phase have not yet been elucidated. High-throughput gene profiling provides an additional unparallel window to study the interface between disease-related events and biological processes. Gene expression studies allow the investigation of hundreds to thousands of genes simultaneously with analysis of complex gene expression changes that are related to treatment.

In the current study we aimed to identify peripheral blood gene transcripts that are specifically up- or downregulated by GA treatment in order to better understand the molecular effects of GA in MS and identify treatment related markers.

\section{Subjects and methods}

\subsection{Study design}

Prospective, randomized. Sheba Medical Center IRB Committee and the Committee for Human Studies, the Israeli Ministry of Health, approved the study. Demographic and clinical variables were obtained from the Sheba Multiple Sclerosis Center Computerized Database Registery.

\subsection{Subjects}

Patients followed at the MS Center, Sheba MC, TelHashomer, Israel, were eligible to partcipate in the study according to the following inclusion criteria:

(1) A relapsing-remitting MS with at least 2 relapses in the year preceding the study and neurologically stable within the 90 days prior to blood withdrawn; (2) No immunomodulatory or immunosuppressive treatment at any time and no steroid treatment for at least 90 days, prior to blood withdrawn; (3) Initiation of Glatiramer Acetate $\left(\mathrm{GA}^{\circledR}, 20 \mathrm{mg}\right.$, subcutaneous daily injections) treatment within the following 14 days after blood withdrawn; (4) Signed written informed consent.

For the qRT-PCR and FACS verification experiments additional samples were obtained from RRMS patients either untreated or treated with GA for at least 6 months, $12 \mathrm{~h}$ after the last GA injection.

For the study of GA gene-expression profiles 26 RRMS patients, mean age $35.2 \pm 3.3$ years, F/M ratio $18 / 8$, disease duration $6.1 \pm 2.9$ years, neurological disability by EDSS $2.2 \pm 0.3$ and annual relapse rate $1.2 \pm 0.4$, before and at 3 months after initiation of GA treatment were enrolled. Samples from 14 patients were used to identifiy GA induced gene expression signature and samples from 12 patients were used for external validation.

For verification of GA induced key genes, by qRTPCR and FACS additional samples were obtained from a group of 20 RRMS patients (10 GA-treated for at least 6 months, $12 \mathrm{~h}$ after the last GA injection, and 10 untreated), mean age $36.1 \pm 3.6$ years, F/M ratio $12 / 8$, disease duration $6.7 \pm 1.3$ years, EDSS $1.7 \pm 0.6$, and annual relapse rate $1.1 \pm 0.2$.

\subsection{RNA isolation and microarray expression profiling}

Peripheral blood mononuclear cells (PBMC) were isolated at baseline and at three months of GA treatment on ficoll - hypaque gradient, total RNA was extracted with trizol reagent (Invitrogen, Carlsbad, CA), and the derived cRNA samples were hybridized to Affymetrix Human Genome U133 A- 2Array containing 18,400 gene transcripts that correspond to 14,500 well annotated human genes (Affymetrix, Inc, Santa Clara, CA). All samples were tested for RNA quality control by $1 \%$ polyacrylamide gel electrophoresis according to Affymetrix protocol.

\subsection{Filtration and normalization}

Prior to microarray analysis all probe sets were filtered to effectively dissociate actual gene expression values from experimental noise. Transcripts that passed noise filtration had $>90 \%$ detection rate across all samples. Expression values were computed from raw CEL files using the Robust Multi-array Average (RMA) model of probe-specific correction algorithm. The corrected probe values were then subjected to quantile normalization to equalize signal intensity across all arrays and the resulting expression values were $\log _{2^{-}}$ transformed. 


\subsection{Data analysis}

Statistical data analysis was performed using the Scoregene software (http://compbio.cs.huji.ac.il/ scoregenes/). To identify GA-treatment induced altered genes the classic parametric t-test, and the nonparametric tests; the threshold number of misclassifications (TNoM) and Info test were applied. TNoM score counts the number of classification errors that occur between compared groups for each gene of the dataset. The best threshold (TNoM=0) implies that no errors have been counted and the distinction between the analyzed groups according to the expression level of a specific gene is maximal. Info score is a defined version of TNoM that measures the misclassifications made by a simple threshold in terms of the information lost (or entropy) of the labels of samples in each side of the threshold. Most informative genes (MIGs) were defined as those that passed a p-value threshold of 0.05 in all three statistical tests. Two-way unsupervised hierarchical clustering analysis was applied to demonstrated differences in gene expression before and after treatment. Fold-change analysis was used to identify genes with expression ratios that differed before and after GA treatment usin Log2(ratios) to compare intensity values.

\subsection{Dual validation strategy}

Internal validation of GA-related gene expression signature was performed by overabundance analysis that examines whether the number of genes in the observed results, at different $p$ values, is as expected under the null hypothesis that the separation of the samples is random, and by LOOCV (Leave One Out Cross Validation) analysis that tests the predictive power of the gene expression signature. The procedure simulates removal of a single sample every trial and trains on the rest until each sample is left out once and the number of correct and incorrect predictions is counted for different $\mathrm{p}$ value thresholds. External validation was performed in an additional set of microarray samples by forward feature selection algorithm implemented in MATLAB, permutations of samples were repeated 50 times and an average error rate for each combination of genes was calculated.

\subsection{Biological functional analysis}

Gene functional annotation, enrichment analysis and pathway reconstruction were performed using David Bioinformatics Resources http://david.abcc.ncifcrf.gov /home.jsp/, Ingenuity Pathways Analysis software, www.ingenuity.com/ and KEGG database. Most significant biological pathways were evaluated using Genomica software http://genomica.weizmann.ac.il, where the gene expression data of MIGs was taken as an input, and the outputs of co-regulated genes were defined as functional modules. Each module reflects the genes that participate in a specific biological process as it consists of the genes whose expression profile corresponds to the signature of the cluster. Modules were statistically analyzed for enrichment of biological processes. Enrichment was defined as the higher proportion of genes in a given gene-set than expected by chance. The cut-off statistical significance for functional module analysis was at $p<0.01$, and for enrichment analysis at $p<0.05$. Based on these findings, GA-induced biological convergent mechanisms operating in RRMS were reconstructed.

\subsection{Microarray data sharing and annotation}

Microarray data and required information on experimental design, sample preparation, data transformation and normalization are reported according to data annotation and exchange guidelines http:/www.mged.org.

\subsection{Verification}

qRT-PCR, Western blot and FACS analyses were applied for verification. qRT-PCR was carried out on 7500 Real-time PCR Cycler (Applied Biosystem, CA,USA) using Taqman probes adjusted to each of the five following MIGs: DR3-HS00237056 m, DR6HS00205419_m, DAPK3-HS00234480 m, CD40HS00386848 $\mathrm{m}$ and TCF8-HS00232783 m. Analysis was carried out by 7500 System W1.2.2 software. GAPDH expression levels were used as internal control for sample normalization.

For FACS analysis PBMC were isolated on ficollhypaque gradient, $3 \times 10^{6}$ cells were fixed by PBS4\%PFA, permeabilized by $0.5 \%$ Tween- 20 (Sigma), washed with PBS and stained for 1 hour, at $4{ }^{\circ} \mathrm{C}$, with $50 \mu \mathrm{l}$ diluted protein concentration $\geqslant 0.1 \mu \mathrm{g} / \mathrm{ml}$ FITCconjugated (Becton Dickinson, USA) monoclonal antibodies for DAPK (Biosciense, USA), DR3 and DR6 (Serotec,UK), CD-40 (Biolegend, USA), and TCF-8 

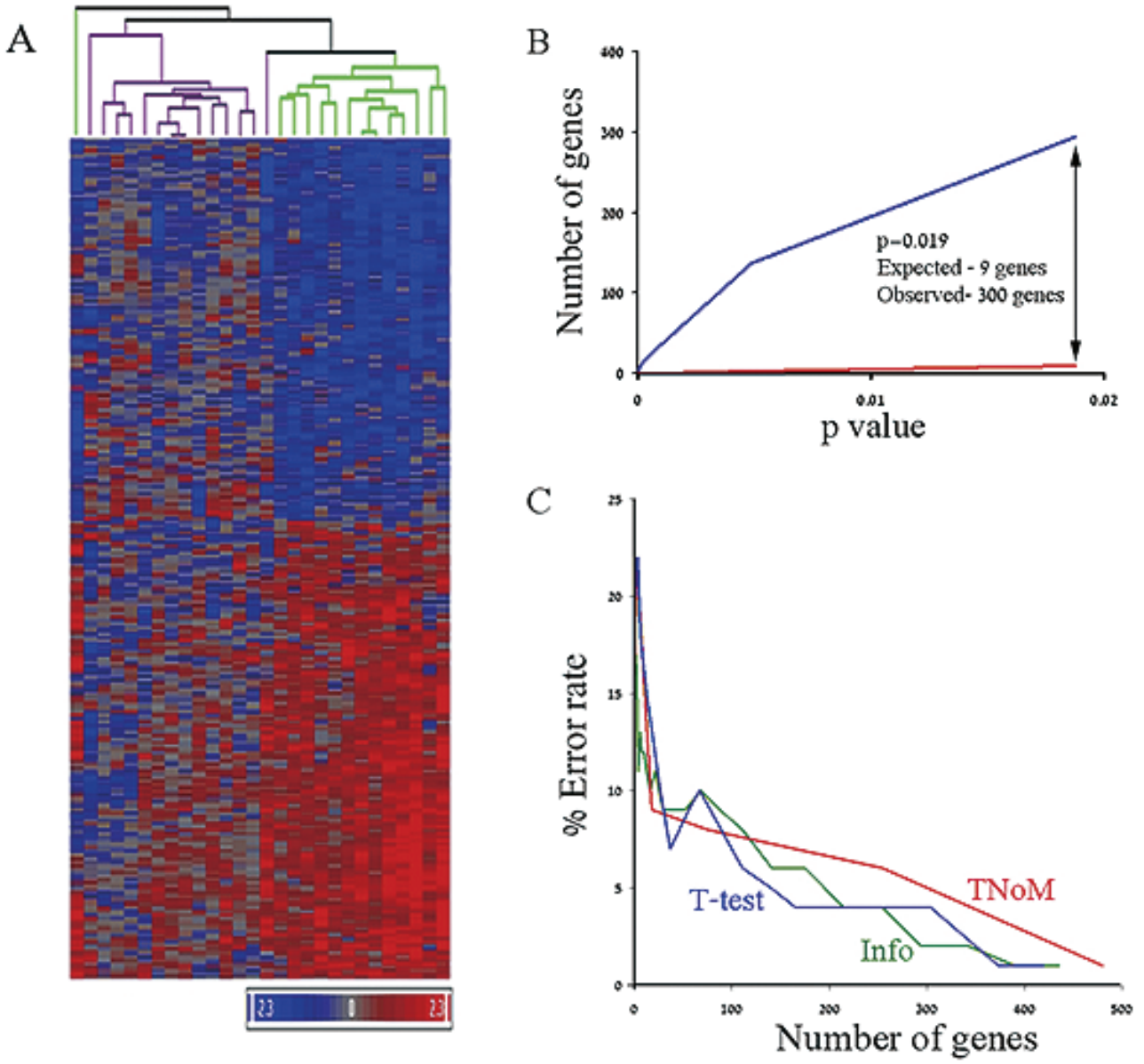

Fig. 1. GA induced gene expression signature. (A) Unsupervised hierarchical clustering of 480 genes that distinguished RRMS patients before (green dendrogram) and at 3 months of GA treatment (violet dendrogram). Each row represents a gene, each column - a patient. Red color indicates an over-expressed gene, blue color indicates a down-expressed gene. The degree of color saturation corresponds to gene expression level shown at the bottom of the image. (B) Overabundance analysis of the actual and expected number of genes that significantly distinguish GA effects at 3 months of treatment. The $x$ axis denotes the number of genes and the $y$ axis shows the $p$ value. The actual number of genes is demonstrated by the blue line, and the expected number of genes by the red line. At $p=0.019$ the expected number of genes is 9 and the observed number of genes is 300. (C) Leave-One-Out-Cross-Validation (LOOCV) classification graph. The $\mathrm{x}$ axis denotes the number of genes and the y axis denotes the error rate in percentages. Using 360 genes (t-test- blue line, Info-green line) and 480 genes (TNoM-red line), an error rate of $3.5 \%$ (one classification error) was demonstrated.

(Aviva biosystems, USA). After staining, $500 \mu \mathrm{l}$ PBS was added to each sample and the percentage of positive cells for each antibody staining was determined (Becton Dickinson, USA). Western blot analysis was performed for MHC class I HLA-A/B/C gene and for NKAT (common subunit of natural killer-associated transcript) gene. Protein samples were prepared using a $10 \%$ acryl-amide SDS-PAGE gel, transferred to nitrocellulose membrane and mouse monoclonal antihuman-HLA-A/B/C (46 kDa), and mouse monoclonal anti-human-NKAT (38 kDa), SantaCruz Biotech Inc, USA, were used as primary antibodies; goat anti-mouse IgG-HRP conjugated (Jackson, West Grove, PA) were applied as secondary antibody.

\section{Results}

\subsection{GA-induced gene expression molecular signature}

GA induced a differential expression of 480 MIGs following a three months treatment period; 262 genes were over-expressed, and 218 genes were downexpressed, suppl Table 1. Unsuppervised hierarchical clustering disclosed two distinquished clusters related to GA treatment effects with only one classification er- 
A

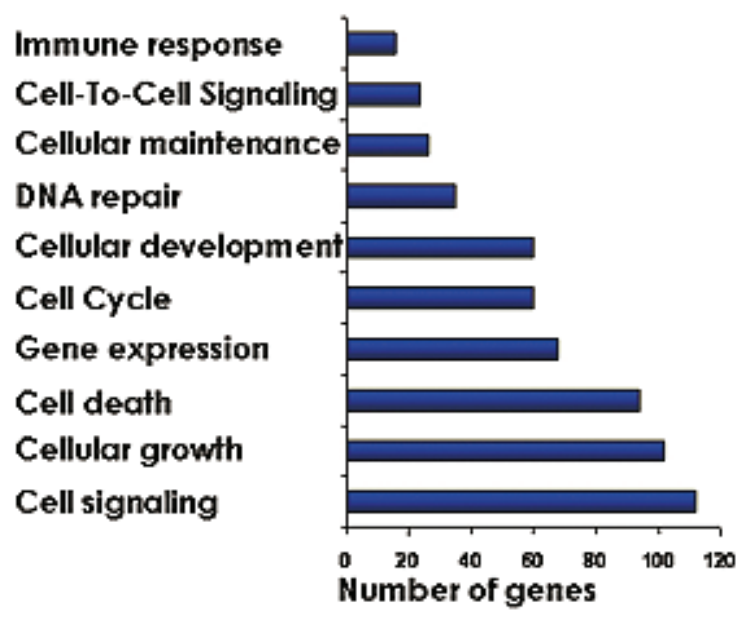

B

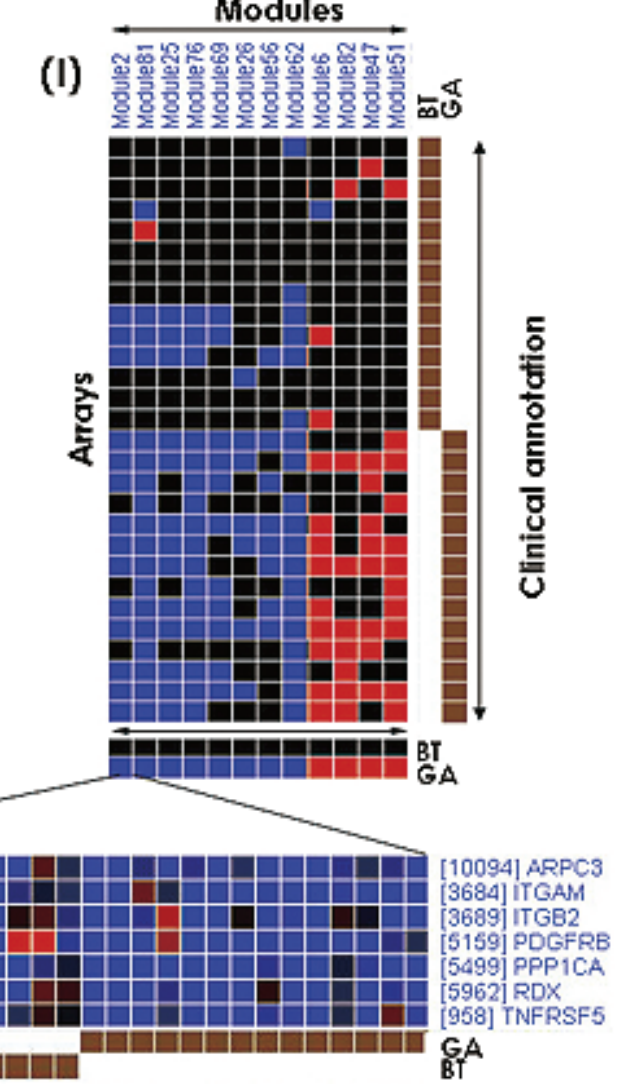

Fig. 2. Biological annotations of GA-induced gene expression signature. (A) Functional. (B) Module analysis of clusters of co-expressed genes across all arrays. (I) The upper graphical panel is a matrix of modules versus arrays where a colored entry indicates that the genes in that module had significantly changed in a coordinated fashion in the respective array. Red color - increased expression, blue - decreased expression, black no change. The right graphical panel shows individual clinical conditions: BT - arrays before treatment; GA - after 3 months of GA treatment. The bottom panel shows the clinical condition for which the modules were significantly enriched. (II) Module \#2 represents suppression of adhesion in response to GA treatment. Red color denotes over-expressed genes, blue - down-expressed.

ror per group, Fig. 1A. Internal validation by overabudance analysis demonstrated significantly higher number of observed $(\mathrm{N}=300)$ than expected $(\mathrm{N}=9)$ genes, Fig. 1B. LOOCV resulted in $3.5 \%$ error rate (one classification error) using 360 genes (t-test and Info test) and 480 genes (TNoM), Fig. 1C. External validation performed by applyig the forward feature selection algorithm to the $480 \mathrm{GA}$-induced MIGs in an independent group of 12 RRMS patients, resulted in 6 gene combinations (each includes 3 to 8 genes) with no classification errors. Using up to 274 gene combinations (each includes 1 to 280 genes) resulted in a maximal average error rate of $20 \%$. These results are highly significant and strongly suggest that the identified GA-related signature is unbiased and represents a large number of target genes, allowing biological analysis of GA-induced regulatoy networkd in RRMS.

\subsection{Biological functional analysis}

Global ontology analysis of the 480 GA-treatment induced MIGs signature demonstrated that these genes extend across a variety of biological processes including cell growth and proliferation, cell cycle, cell death, cell signaling and immune response mechanisms, Fig. 2A.

Functional module analysis of joint behavior of clusters of co-expressed genes across all arrays identified 12 statistically significant modules, Fig. 2B (I), that included between 7 to 143 genes; 4 modules mainly contained up-regulated genes and 8 modules mainly contained down-regulated genes. An example of module that represents suppression of adhesion in response to GA treatment is demonstrated in Fig. 2B(II). Module enrichment demonstrated significant over-presentation of genes related to apoptosis $(p<0.0007)$, apopto- 
(a)

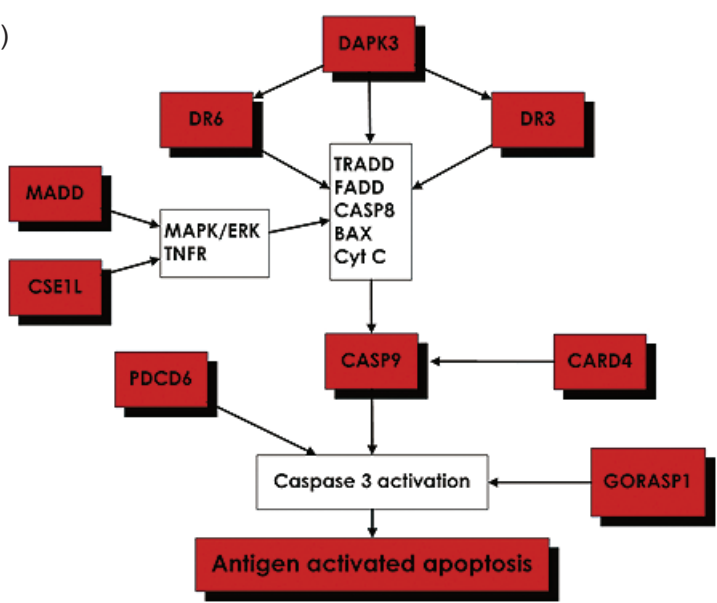

(b)

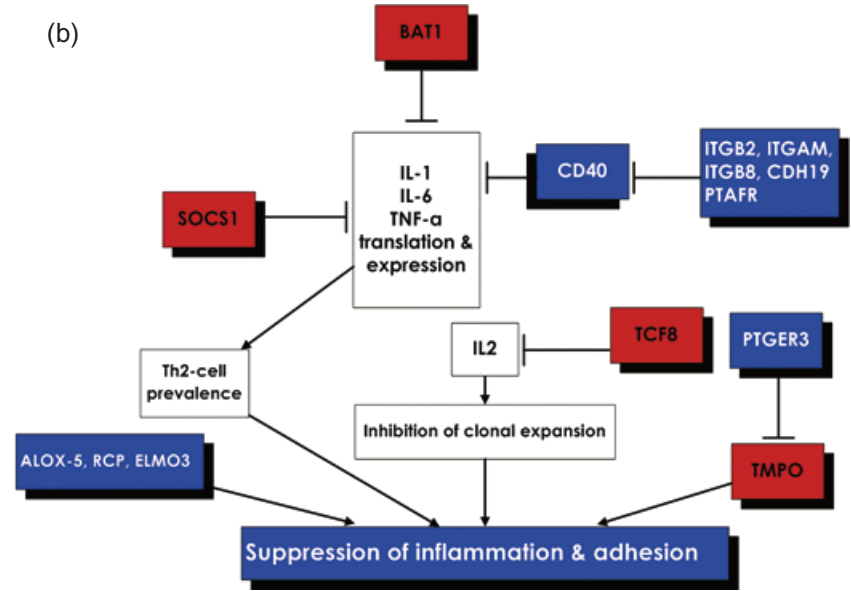

(c)

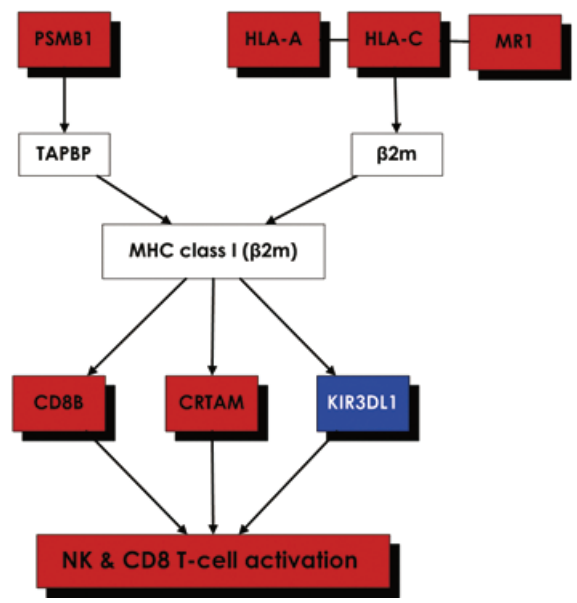

Fig. 3. GA-induced biological convergent mechanisms operating in RRMS. (A) Apoptosis (B) Inflammation and adhesion (C) MHC class I antigen presentation pathways. Red color - over-expressed genes, blue - down-expressed. 
sis regulation $(p<0.01)$, and antigen presentation $(p<0.05)$ in modules combined mainly from overexpressed genes. Genes related to adhesion and inflammatory mechanisms were enrichred ( $p<0.01$, for both) in the down-expressed modules. Accordinly, we reconstructed GA-induced immunological pathways related to the most significant biological processes in GA induced gene expression signature: apoptosis, antigen presentation, adhesion and inflammation.

\subsection{GA-induced biological convergent processes operating in RRMS}

(1) Apoptosis. GA-induced apoptotis related pathway, Fig. 3A, is mainly up-regulated and includes death associated protein kinase 3 (DAPK3) that mediates apoptosis through death receptor 3 (DR3) and death receptor 6 (DR6) both also found to be overexpressed. DR3 is involved in removal of self-reactive $\mathrm{T}$ cells in the thymus [9], and DR6 plays a significant role in the activation and differentiation of $\mathrm{T}$ helper cells [10]; Both DR3 and DR6 operate through TNFR1 -associated death domain protein (TRADD) to induce apoptosis and activation of nuclear factor kappaB. DR3 signal transduction is mediated by a complex of intracellular signaling molecules including TRADD, TRAF2, FADD, and FLICE [11]. Additional identified MIGs include MAP-kinase activating death domain (MADD) that also interacts with TNFR-1 and activates mitogen-activated protein kinase [12]; Programmed cell death 6 (PDCD6) that encodes $\mathrm{Ca}(2+)$-binding protein essential for T-cell receptor and Fas-induced cell death, interacts with death-associated protein kinase 1 (DAPk1) and affects apoptosis via caspase-3 dependent pathway [13]; Caspase 9 (CASP9) that contributes to caspase-3 activation [14]; Caspase recruitment domain family, member 4 (CARD4) that is essential for CASP9 activation, as well as to promote apoptosis [15]; Ubiquitin-activating enzyme E1-like protein (UBE1L), a member of the ubiquitin-activating gene family that triggers apoptosis [16]; Cellular apoptosis susceptibility gene (CSE1L/CAS) that is necessary for TNF-a induced apoptosis [17], and caspase 3 substrate golgi associated protein GRASP65 (GORASP) that is critical for cell cycle regulation, growth and apoptosis [18].

(2) Inflammation. GA treatment induced pathway of suppression of inflammation, Fig. 3B, includes overexpression of genes that function as negative regulators of inflammation ilike HLA-B associated transcript 1(BAT1) [19]; Transcription factor 8 (TCF8) that sup- press IL2 expression [20], and suppressor of cytokine signaling 1 (SOCS1) that operates as a negative regulator of JAK-STAT cascade [21]. In accordance, GA down-regulated genes related to inflammatory pathways including tumor necrosis factor receptor superfamily molecule, member 5 (CD40, TNFRSF5) that is expressed on B cells, monocytes and dendritic cells and mediates a broad variety of immune and inflammatory responses like immunoglobulin isotype switching, germinal center formation and stimulation of the humoral memory response [22]; Arachidonate 5-liphoxigenase (ALOX5) expressed by macrophages and known to be involved in inflammation and immediate hypersensitivity [23] ; Rab coupling protein (RCP) known to be involved in phagocytosis [24], and low density lipoprotein receptor-related protein (LRP1) that acts as a sensor for necrotic cell death in tissues leading to proinflammatory immune responses [25].

(3) Adhesion. The group of genes encoding integrins and adhesion molecules that are down-regulated by GA, Fig. 3B, include leukocyte cell adhesion molecule CD18 (ITGB2) and integrin beta 8 (ITGB8) that are associated with leukocyte adhesion [26]; Human leukocyte adhesion glycoprotein Mac-1(ITGAM) responsible for adherence to endothelium [27]; Platelet activating factor receptor (PTAFR) that mediates inflammation, allergy and septic shock [28]; Cadherin 19 (CDH19) that acts as moderator of selective cell-cell adhesion [29], and engulfment and cell motility gene 3 (ELMO3 ) involved in cell migration [30].

(4) Antigen presentation. GA treatment up-regulated several MHC class1 antigen presenting molecules, Fig. 3C. These include HLA-A and HLA-C that present endogenous antigens via MHC class I related proteins; T-cell glycoprotein CD8B (CD8B1) expressed only on cytotoxic $\mathrm{T}$ cells that recognize antigen in the context of MHC class 1 [31]; MHC class 1 restricted $\mathrm{T}$ cell-associated molecule (CRTAM) that is induced in activated $\mathrm{CD}^{+} \mathrm{T}$ cells and in a subset of $\mathrm{CD}^{+}{ }^{+} \mathrm{T}$ cells and interacts with nectin- 2 (Necl2) to promote NK cell cytotoxicity towards otherwise poorly immunogenic targets [32]. In $\mathrm{CD}^{+} \mathrm{T}$ cells CRTAM-Necl-2 interactions lead to increased secretion of IFN- $\gamma$ and IL-22. In agreement with these findings, immunoproteasome (PSMB1) involved in the presentation of endogenous antigens by MHC class I molecules [33] was up-regulated, and killer cell immunoglobulin-like receptor-three domains-long cytoplasmic tail-1(KIR3DL1) that regulates the inhibition and activation of NK cell responses through recognition of MHC-class I molecules on target cells [34] was down-regulated. 
A

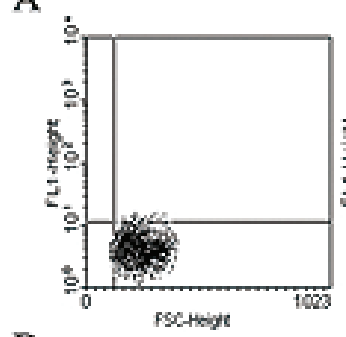

B

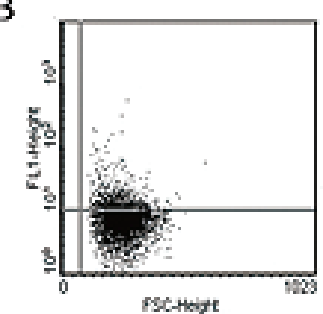

C

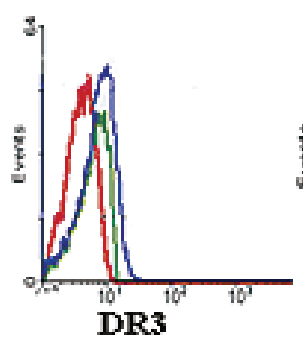

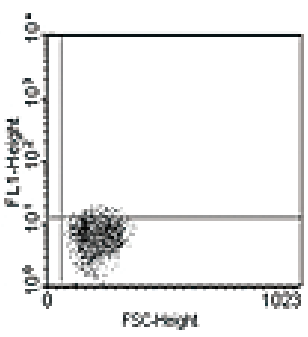

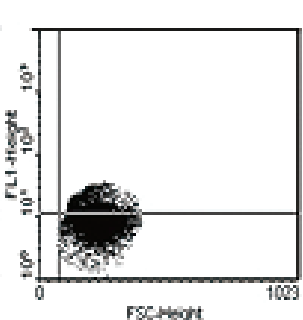

Fscreopt

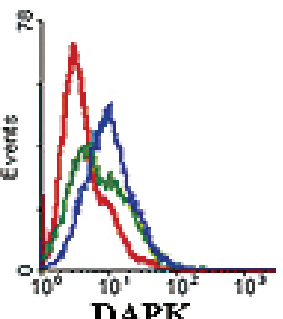

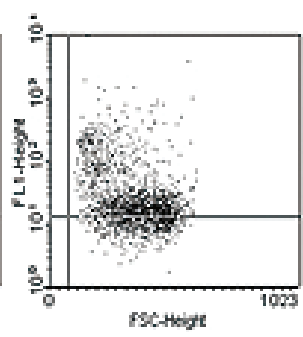

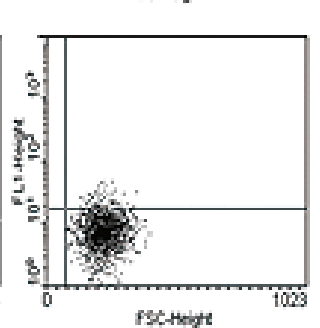

pecthent

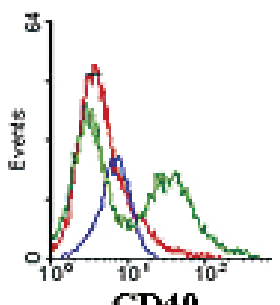

CD40
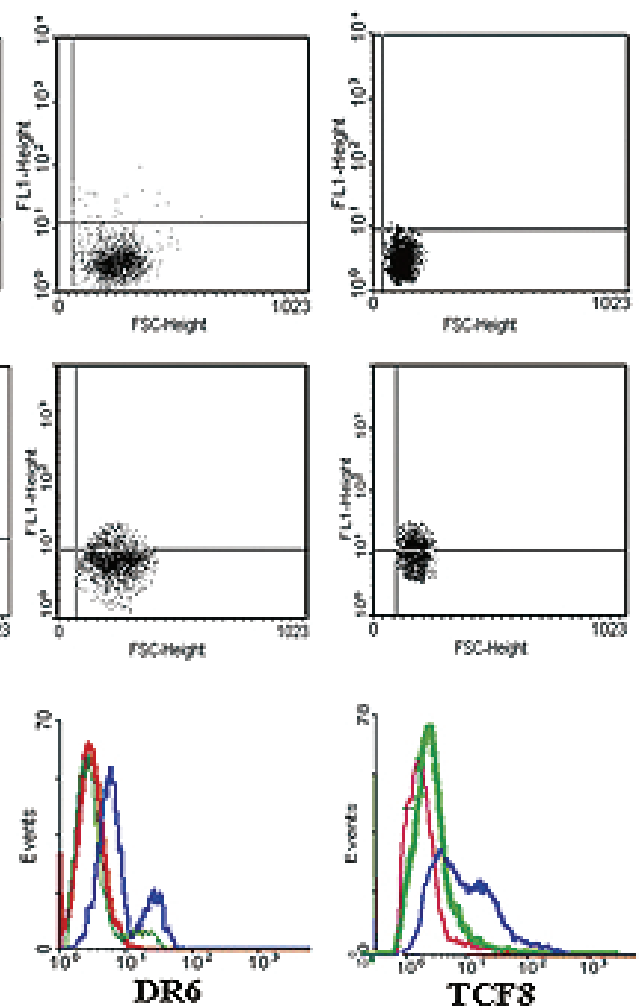

Fig. 4. Verification of key GA-induced genes at the protein level by FACS cytometry analysis. (A) PBMC from an untreated MS patient. (B) PBMC from a GA-treated patient. The number of cells at each intensity is shown by the number of dots where each dot represents a single cell. X-Axis: forward scatter of cell size parameters; Y Axis: antibody-conjugated color excitation. (B) Colored histograms of fluorescent intensity of GA-treated (blue), untreated (green) and internal control without antibody (red). X-axis: level of fluorescence emitted by the labeled cells; Y-axis: number of cells.

Verification: The expression of five key genes: DR3, DR6, CD40, DAPK3, and TCF8 within the GA-induced MIGs expression signature was verified by qRT-PCR. The results correlated with the microarray findings demonstrating corresponding supression/activation at the mRNA level in GA treated (N $=10)$ as compared to untreated $(\mathrm{N}=10)$ RRMS patients; DR3 $(p=0.0057)$, DR6 $(p=0.01), \operatorname{CD} 40(p=$ $0.0002)$, DAPK3 $(p=0.004)$ and TCF8 $(p=0.01)$. FACS analysis similarly demonstrated a corresponding supression/activation at the protein level in GA treated $(\mathrm{N}=10)$ as compared to untreated $(\mathrm{N}=10)$ RRMS patients; DR3 (fold change $=2.97, p=0.002$ ), DR6 (fold change $=4.6, p=0.009$ ), $\mathrm{CD} 40$ (fold change $=-$ $3.39 p=0.007$ ), DAPK3 (fold change $=4.43, p=$ 0.003 ) and TCF 8 (fold change $=4.15, p=0.007$ ). The results of the FACS analysis are illustrated in Fig. 4.

The protein expression of the key genes of MHC class I pathway HLA-A/B/C and the Natural Killer associated gene NKAT gene were confirmed by Western blot, Fig. 5.

\section{Discussion}

In the current study we identified a specific GA induced gene expression molecular signature that contains 480 MIGs extending across a variety of biological processes like cell growth and proliferation, cell cycle, cell death, cell signaling and immune response mechanisms. This gene expression signature was validated by advanced bioinformatic analyses and significant genes were further verified in an independent cohort of patients at the mRNA and protein level demonstrating that the identified GA-related signature is robust and biologically significant. Reconstruction of GA-induced pathways operating in the early treatment phase in RRMS demonstrated convergent biological mechanisms related to apoptosis, inflammation, adhesion and MHC class I antigen presentation. MS pathogenesis involves an abnormal $\mathrm{T}$ cells mediated autoimmune response to neural antigens, leading to mononuclear cell recruitment, oligodendrocyte loss, demyelination and axonal degeneration. The persistence of these autoreac- 
A

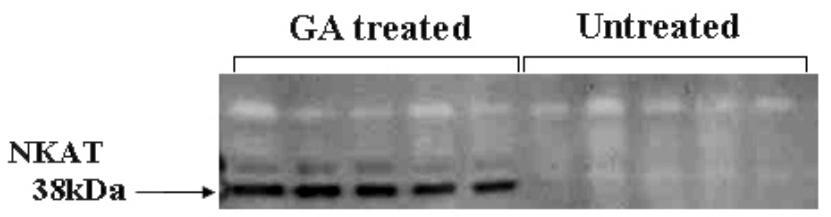

B

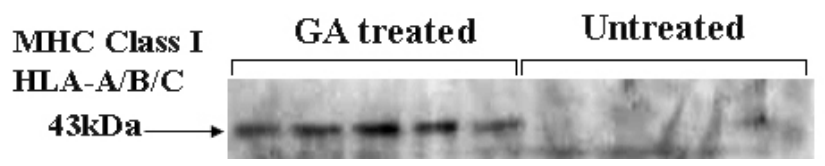

Fig. 5. Verification of key GA-induced genes at the protein level by Western Blot. (A) MHC class I pathway HLA-A/B/C. (B) Natural Killer associated transcript NKAT.

tive $\mathrm{T}$ cells reflects impairment in apoptosis pathways in MS, and over-expression of the apoptosis inhibitor FLIP in T cells correlates with disease activity [35]. In the current study we demonstrated that GA treatment induced up-regulation of apoptotic-related genes like DAPK3, DR3, and DR6 and enhanced regulatory effects on peripheral lymphocytes through activation of pro-apoptotic genes like PDCD6, CASP9, CARD4 and GORASP1. We also demonstrated over-expression of MADD and CSE1L that bind to the death domain of TNFR1 and activate the MAP kinase cascade [36]. In accordance to our findings, GA treatment was reported to induce the regulation of apoptosis at the protein level by increasing the ratio of pro/anti apoptotic molecules BAX/BCL-2 [37], and to increase the apoptosis of $\mathrm{CD}^{+} \mathrm{T}$ cells [38]. Our findings further support the restoration of impaired apoptosis by GA and detail the particular molecules involved.

The inflammatory response known to occur in MS was also affected by GA treatment. The identified overexpression of BAT1 that down-regulates the acute phase cytokines IL1, IL6 and TNF- $\alpha$ production [19] and explains the Th1/Th2 shift known to result under GA treatment [39]. In concert with the over-expression of BAT1, the down-expression of CD40, a member of the TNFR family that also inhibits the expression of IL1, IL6 and TNF- $\alpha$ additionally contributes to GA-induced Th2 shift [40]. Over-expression of SOCS, a member of the feedback control mechanisms that regulate cytokine signal transduction through the JAK/STAT pathway [41], leads to additional suppression of inflammatory cytokines and increases Th2 cell prevalence. SOCS1 was also reported to suppress autoimmunity and protect myelinating oligodendrocytes from inflammation by inhibiting IFN-gamma [42]. Additional pathway associated with GA suppression of inflammation is related to over-expression of TCF8 that inhibits IL-2 which is essential for T-cell proliferation and differentiation. GA treatment also suppressed non-specific inflammatory response inducing over-expression of TMPO followed by PTGER3 inhibition, and down-regulating the inflammatory genes ALOX-5 expressed by macrophages, RCP gene that plays an important role in phagosome maturation, and ELMO3 that is involved in cytoskeletal rearrangements during phagocytosis of apoptotic cells [23,24,30]. All together these findings demonstrate that GA induced specific suppression of T-cell and macrophage mediated inflammation as well as decreaed non-specific inflammatory responses. In context with the suppression of inflammatory pathways, repression of adhesion molecules demonstrated by down regulation of CD40 shut-down the integrin signaling cascade and further suppress autoreactive T cell activation [43].

Finally, the innovative GA-related pathway we identified in the current study, applies to antigen presentation through MHC class I. The inhibitory effects of GA on the induction of MHC class II molecules specifically HLA-DR and HLA-DQ are well known [44]. However, the stimulation of immunologic activity and inflammation through MHC class I molecules, specifically HLA-A and HLA-C, are first reported. GA treatment is known to upregulate $\mathrm{CD}^{+} \mathrm{T}$ cells that respond to diverse antigents displayed by MHC class- 1 molecules [45]. This suggest that at least part of the early immunomodulatory effects of GA are mediated by GA-induced MHC class I-restricted CD8 ${ }^{+} \mathrm{T}$ cells upregulation. As $\mathrm{CD}^{+}$cells have divergent roles in MS, we suggest that GA mainly induces expression of $\mathrm{CD}^{+}$Treg that in turn by secreting regulatory cytokines such as IL-10 and TGF- $\beta$, down-regulate the autoreactive T-cell response [46]. Additionally, the over-expression of MHC class I molecules induced by GA treatment can augment NK cell activity. Indeed, 
increase in NK cells was reported to be associated with decreased MS disease activity [47]. In the same trend of concept, GA treatment in an animal model of hepatic fibrosis, demonstrated an antifibrotic effect associated with increased NK cells [48], suggesting a possible role of NK cells in regulation of the autoimmune inflammatory process in MS. To summarize, the convergent biological pathways of GA early treatment effects orchestrate to immunomodulate and decelerate the autoimmune processes operating in RRMS. The main mechanisms involved include stimulation of apoptosis combined with suppression of T-cell inflammatory and adhesion responses and the enhancement of MHC class I antigen presentation that can induce $\mathrm{NK}$ and $\mathrm{CD} 8^{+} \mathrm{T}$ cell regulatory activities.

\section{Supplementary Data}

Supplementary Table 1 can be viewed on: http://eng. sheba.co.il/542/.

\section{References}

[1] L. Steinman, R. Martin, C. Bernard, P. Conlon and J.R. Oksenberg, Multiple sclerosis: deeper understanding of its pathogenesis reveals new targets for therapy, Annu Rev Neurosci 25 (2002), 491-505.

[2] B. Hemmer, S. Cepok, S. Nessler and N. Sommer, Pathogenesis of multiple sclerosis: an update on immunology, Curr Opin Neurol 15 (2002), 227-231.

[3] J.S. Wolinsky, Glatiramer acetate for the treatment of multiple sclerosis, Expert, Opin Pharmacother 5 (2004), 875-891.

[4] F. Martinelli Boneschi, M. Rovaris, K.P. Johnson, A. Miller, J.S. Wolinsky, D. Ladkani G. Sheroni C. Giancarlo and F. Massimo, Effects of glatiramer acetate on relapse rate and accumulated disability in multiple sclerosis: meta-analysis of three double-blind, randomized, placebo controlled clinical trials, Mult Scler 9 (2003), 349-355.

[5] R. Arnon and R. Aharoni, Mechanism of action of glatiramer acetate in multiple sclerosis and its potential for the development of new applications, Proc Natl Acad Sci USA, 101(Suppl 2) (2004), 14593-14598.

[6] R. Arnon and R. Aharoni, Neurogenesis and neuroprotection in the CNS-fundamental elements in the effect of Glatiramer acetate on treatment of autoimmune neurological disorders, Mol Neurobiol 36 (2007), 245-253.

[7] R. Aharoni, A. Herschkovitz, R. Eilam, M. Blumberg-Hazan, M. Sela, W. Bruck and R. Arnon, Proc Natl Acad Sci USA 12 (2008), 11358-11363.

[8] J.M. Scorisa, R.G. Zanon, C.M. Freria and A.L. de Oliveira, Glatiramer acetate positively influences spinal motoneuron survival and synaptic plasticity after ventral root avulsion, Neurosci Lett 451 (2009), 34-39.

[9] E.C. Wang, A. Thern, A. Denzel, J. Kitson, S.N. Farrow and M.J. Owen, DR3 Regulates negative selection during thymocyte development, Mol Cell Biol 21 (2001), 3451-3461.
[10] H. Zhao, M. Yan, H. Wang, S. Erickson, I.S. Grewal and V.M. Dixit, Impaired c-Jun amino terminal kinase activity and $\mathrm{T}$ cell differentiation in death receptor 6-deficient mice, $J$ Exp Med 194 (2001), 1441-1448.

[11] A.M. Siwkowski, L.A. Madge, S. Koo, E.L. McMillan, B.P. Monia, J.S. Pober and B.F. Baker, Effects of antisense oligonucleotide-mediated depletion of tumor necrosis factor (TNF) receptor 1-associated death domain protein on TNFinduced gene expression, Mol Pharmacol 66 (2004), 572-579.

[12] A.M. Al-Zoubi, E.V. Efimova, S. Kaithamana, O. Martinez, El-Idrissi Mel-A, R.E. Dogan and B.S. Prabhakar, Contrasting Effects of IG20 and its Splice Isoforms, MADD and DENN$\mathrm{SV}$, on Tumor Necrosis Factor $\alpha$-induced Apoptosis and Activation of Caspase-8 and -3, J Biol Chem 276 (2001), 4720247211.

[13] J.H. Lee, S.B. Rho and T. Chun, Programmed cell death 6 (PDCD6) protein interacts with death-associated protein kinase1(DAPk1): additive effect on apoptosis via caspase-3 dependent pathway, Biotechnol Lett 27 (2005), 1011-1015.

[14] C. Pop, J. Timmer, S. Sperandio and G.S. Salvesen, The apoptosome activates caspase- 9 by dimerization, Mol Cell $\mathbf{2 2}$ (2006), 269-275.

[15] N. Inohara, T. Koseki, L. del Peso, Y. Hu, C. Yee, S. Chen, R. Carrio, J. Merino, D. Liu, J. Ni, and G. Núñez, Nod1, an Apaf-1-like activator of caspase-9 and nuclear factor-kappa-B, J Biol Chem 274 (1999), 14560-14567.

[16] I. Pitha-Rowe, W.J. Petty, Q. Feng, P.H. Koza-Taylor, D.A. Dimattia, L. Pinder, K.H. Dragnev, N. Memoli, V. Memoli, T. Turi, J. Beebe, S. Kitareewan and E. Dmitrovsky, Microarray analyses uncover UBE1L as a candidate target gene for lung cancer chemoprevention, Cancer Res 64 (2004), 8109-8115.

[17] P. Behrens, U. Brinkmann and A. Wellmann, CSE1L/CAS: Its role in proliferation and apoptosis, Apoptosis 8 (2003), 39-44.

[18] S. Yoshimura, K. Yoshioka, F.A. Barr, M. Lowe, K. Nakayama, S. Ohkuma and N. Nakamura, Convergence of cell cycle regulation and growth factor signals on GRASP65, $J$ Biol Chem 280 (2005), 23048-23056.

[19] R.J. Allcock, J.H. Williams and P. Price, The central MHC gene, BAT1, may encode a protein that down-regulates cytokine production, Genes Cells 6 (2001), 487-494.

[20] T.M. Williams, G. Montoya, Y. Wu, R.L. Eddy, M.G. Byers and T.B. Shows, The TCF8 gene encoding a zinc finger protein (Nil-2-a) resides on human chromosome 10p11.2, Genomics 14 (1992), 194-196.

[21] R. Starr, T.A. Willson, E.M. Viney, L.J. Murray, J.R. Rayner, B.J. Jenkins T.J. Gonda, W.S. Alexander, D. Metcalf, N.A. Nicola and D.J. Hilton, A family of cytokine-inducible inhibitors of signalling, Nature 387 (1997) 917-921.

[22] U. Schonbeck and P. Libby, The CD40/CD154 receptor/ligand dyad, Cell Mol Life Sci 58 (2001), 4-43.

[23] R. De Caterinam and A. Zampolli, From asthma to atherosclerosis- 5-lipoxygenase, leukotrienes, and inflammation, New Eng J Med 350 (2004), 4-7.

[24] M.T. Damiani, M. Pavarotti, N. Leiva, A.J. Lindsay, M.W. McCaffrey and M.I. Colombo, Rab coupling protein associates with phagosomes and regulates recycling from the phagosomal compartment, Traffic 5 (2004), 785-797.

[25] S.N. Maiti, K. Balasubramanian, J.A. Ramoth and A.J. Schroit. Beta-2-glycoprotein 1-dependent macrophage uptake of apoptotic cells. Binding to lipoprotein receptor-related protein receptor family members, J Biol Chem 283 (2008), 37613766.

[26] R.W. Wilson, C.M. Ballantyne, C.W. Smith, C. Montgomery, A. Bradley, W.E. O'Brien, A.L. Beaudet, Gene targeting yields 
a CD18-mutant mouse for study of inflammation, J Immunol 151 (1993), 1571-1578.

[27] M.A. Arnaout, S.K. Gupta, M.W. Pierce and D.G. Tenen, Amino acid sequence of the alpha subunit of human leukocyte adhesion receptor Mo1 (complement receptor type 3), J Cell Biol 106 (1988), 2153-2158.

[28] S. Ishii, T. Nagase and T. Shimizu, Platelet-activating factor receptor, Prostaglandins Other Lipid Mediat 68-69 (2002), 599-609.

[29] M. Pignatelli. Integrins, cadherins, and catenins: molecular cross-talk in cancer cells, J Pathol 186 (1998), 1-2.

[30] T.L. Gumienny, E. Brugnera, A.C. Tosello-Trampont, J.M. Kinchen, L.B. Haney, K. Nishiwaki, S. Walk, M. Nemergut, I. Macara and R. Franci, CED-12/ELMO, a novel member of the CrkII/Dock180/Rac pathway, is required for phagocytosis and cell migration, Cell 107 (2001), 27-41.

[31] D. Kioussis and W. Ellmeier, Chromatin and CD4, CD8A and CD8B gene expression during thymic differentiation, Nat Rev Immunol 2 (2002), 909-919.

[32] K.S. Boles, W. Barchet, T. Diacovo, M. Cella and M. Colonna, The tumor suppressor TSLC1/NECL-2 triggers NK-cell and CD8+ T-cell responses through the cell-surface receptor CRTAM, Blood 106 (2005), 779-786.

[33] Z. Trachtulec, C. Vlcek, O. Mihola and J. Forejt, Comparative analysis of the PDCD2-TBP-PSMB1 region in vertebrates, Gene 335 (2004), 151-157.

[34] M.P. Martin, X. Gao, J.H. Lee, G.W. Nelson, R. Detels, J.J. Goedert, S. Buchbinder, K. Hoots, D. Vlahov, J. Trowsdale, M. Wilson, S.J. O'Brien and M. Carrington, Epistatic interaction between KIR3DS1 and HLA-B delays the progression to AIDS, Nat Genet 31 (2002), 429-434.

[35] Y.K. Semra, O.A. Seidi and M.K. Sharief, Overexpression of the apoptosis inhibitor FLIP in T cells correlates with disease activity in multiple sclerosis, J Neuroimmunol 113 (2001), 268-274.

[36] B.M. Brinkman, J.B. Telliez, A.R. Schievella, L.L. Lin and A.E. Goldfeld, Engagement of tumor necrosis factor (TNF) receptor 1 leads to ATF-2- and p38 mitogen-activated protein kinase-dependent TNF-alpha gene expression, J Biol Chem 274 (1999), 30882-30886.

[37] M. Ruggieri, C. Avolio, S. Scacco, C. Pica, A. Lia, G.B. Zimatore, S. Papa, P. Livrea and M. Trojano, Glatiramer acetate induces pro-apoptotic mechanisms involving Bcl-2, Bax and Cyt-c in peripheral lymphocytes from multiple sclerosis patients, J Neurol 253 (2006), 231-236.

[38] M. Rieks, V. Hoffmann, O. Aktas, M. Juschka, I. Spitzer, N. Brune, S. Schimrigk, H. Przuntek and D. Pöhlau, Induction of apoptosis of $\mathrm{CD} 4+\mathrm{T}$ cells by immunomodulatory therapy of multiple sclerosis with glatiramer acetate, Eur Neurol $\mathbf{5 0}$ (2003), 200-206.

[39] M. Chen, B. Gran, K. Costello, K. Johnson, R. Martin and S. Dhib-Jalbut, Glatiramer acetate induces a Th2-biased response and crossreactivity with myelin basic protein in patients with MS, Mult Scler 7 (2001), 209-219.

[40] A.G. Eliopoulos, M. Stack, C.W. Dawson, K.M. Kaye, L. Hodgkin, S. Sihota, M. Rowe and L.S. Young, Epstein-Barr virus-encoded LMP1 and CD40 mediate IL-6 production in epithelial cells via an NF-kappaB pathway involving TNF receptor-associated factors, Oncogene 14 (1997), 2899-2916.

41] I.D. Dimitriou, L. Clemenza, A.J. Scotter, G. Chen, F.M. Guerra and R. Rottapel, Putting out the fire: coordinated suppression of the innate and adaptive immune systems by SOCS1 and SOCS3 proteins, Immunol Rev 224 (2008), 265-283.

[42] R. Balabanov, K. Strand, A. Kemper, J.Y. Lee and B. Popko, Suppressor of cytokine signaling 1 expression protects oligodendrocytes from the deleterious effects of interferon-gamma, J Neurosci 26 (2006), 5143-5152.

[43] K. Gerritse, J.D. Laman, R.J. Noelle, J.A. Ledbetter, W.J Boersma and E. Claassen, CD40-CD40 ligand interactions in experimental allergic encephalomyelitis and multiple sclerosis, Proc Natl Acad Sci USA 93 (1996), 2499-2504.

[44] A. Ben-Nun, I. Mendel, R. Bakimer, M. Fridkis-Hareli, D. Teitelbaum, R. Arnon, M. Sela and N. Kerlero de Rosbo, The autoimmune reactivity to myelin oligodendrocyte glycoprotein (MOG) in multiple sclerosis is potentially pathogenic: effect of copolymer 1 on MOG-induced disease, J Neurol 243(4 Suppl 1), (1996), S14-S22.

[45] N.J. Karandikar, M.P. Crawford, X. Yan, R.B. Ratts, J.M. Brenchley, D.R. Ambrozak, A.E. Lovett-Racke, E.M. Frohman, P. Stastny, D.C. Douek, R.A Koup and M.K Racke, Glatiramer acetate (Copaxone) therapy induces CD8(+) T cell responses in patients with multiple sclerosis, J Clin Invest 109 (2002), 641-649.

[46] A.J. Johnson, G.L. Suidan, J. McDole and I. Pirko, The CD8 $\mathrm{T}$ cell in multiple sclerosis: suppressor cell or mediator of neuropathology? Int Rev Neurobiol 79 (2007), 73-97.

[47] L. Airas, M. Saraste, S. Rinta, I. Elovaara, Y.H. Huang and H. Wiendl, Immunoregulatory factors in multiple sclerosis patients during and after pregnancy: relevance of natural killer cells, Clin Exp Immunol 151 (2008), 235-243.

[48] A. Horani, N. Muhanna, O. Pappo, A. Melhem, C.E. Alvarez, S.W. Wehbi, K. Dimitrios, S.L. Friedman and R. Safadi, Beneficial effect of glatiramer acetate (Copaxone) on immune modulation of experimental hepatic fibrosis, Am J Physiol Gastrointest Liver Physiol 292 (2007), G628-G638. 


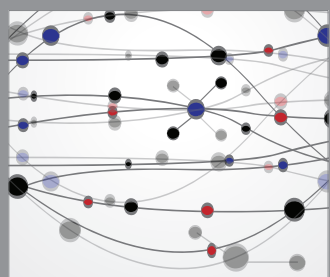

The Scientific World Journal
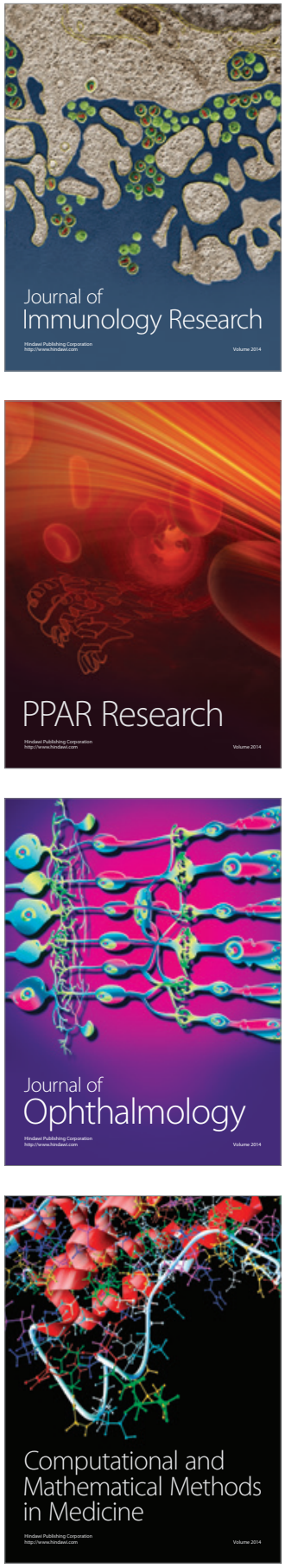

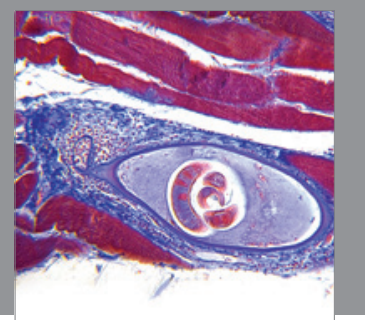

Gastroenterology

Research and Practice
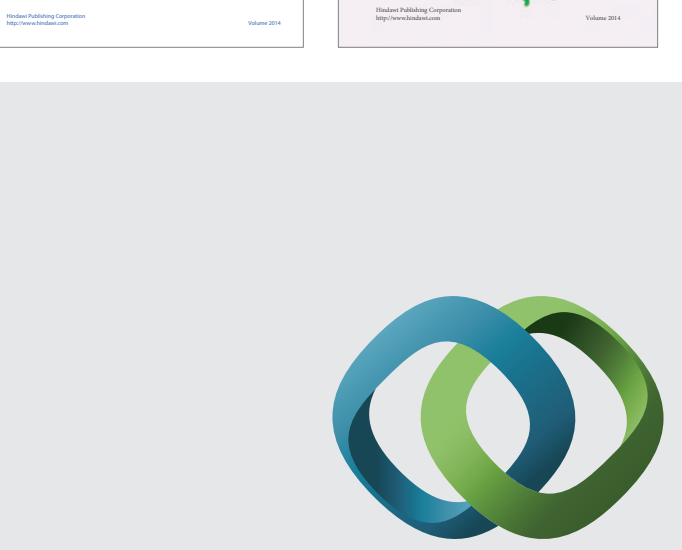

\section{Hindawi}

Submit your manuscripts at

http://www.hindawi.com
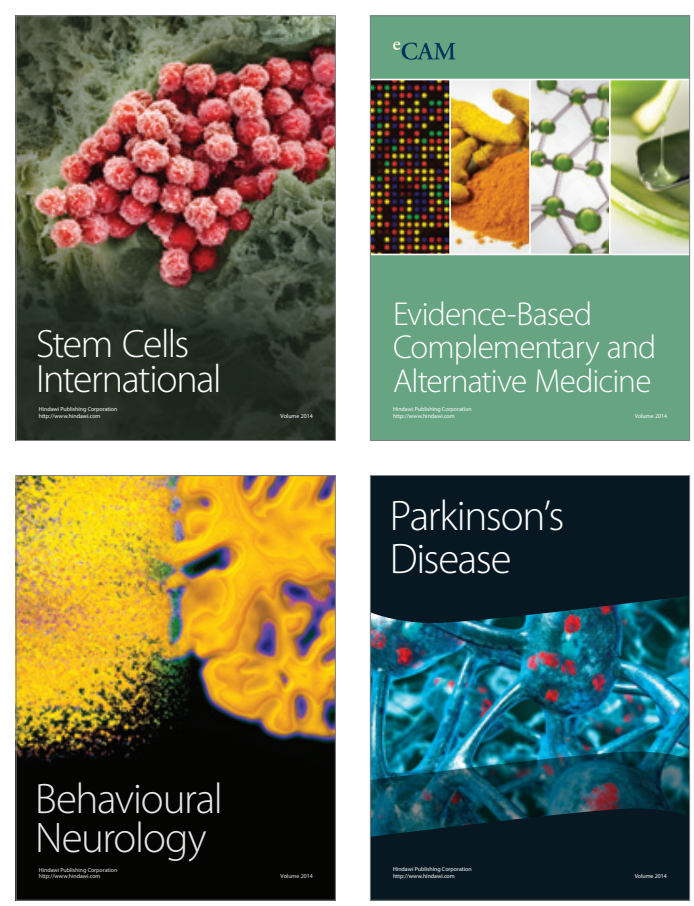

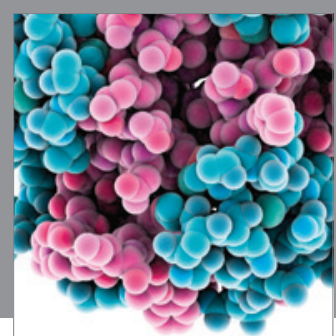

Journal of
Diabetes Research

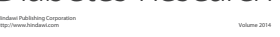

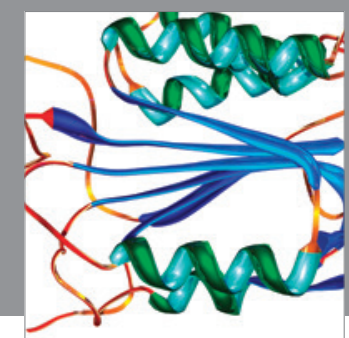

Disease Markers
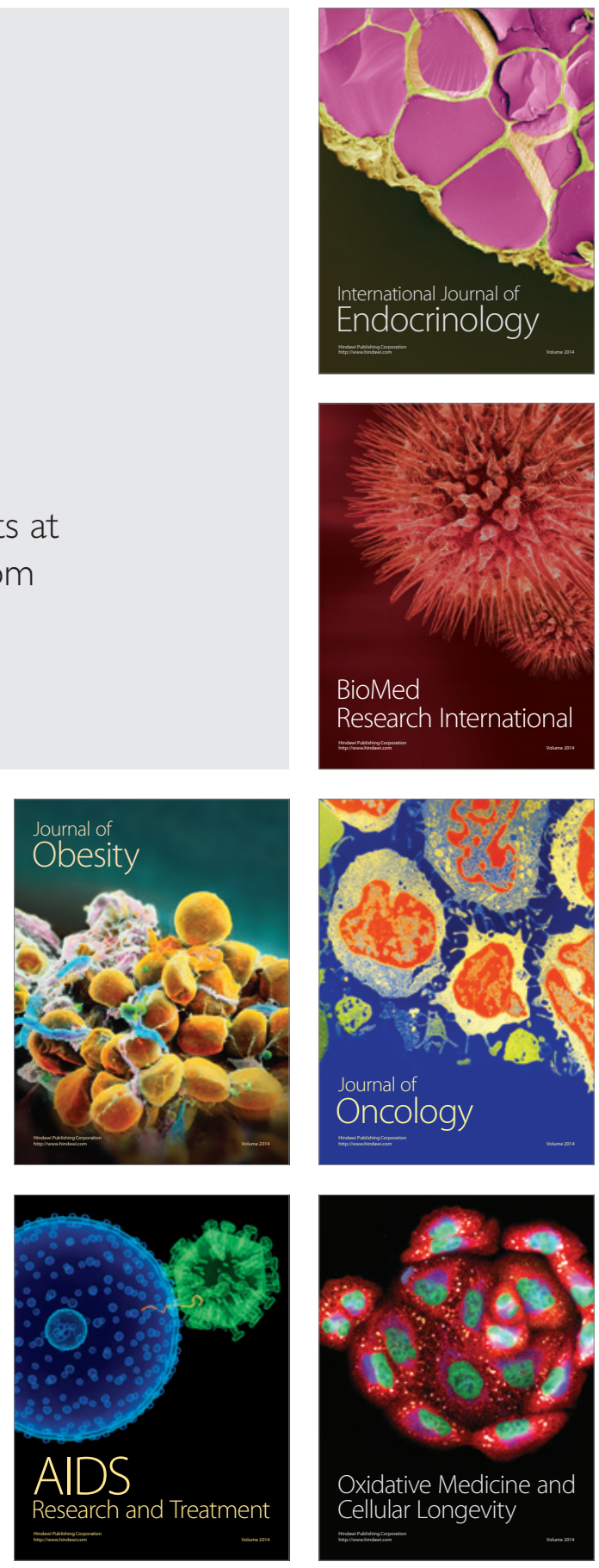